\title{
tic\&société
}

Vol. 7, $\mathrm{N}^{\circ} 1$ | 1er semestre 2013

Formes et enjeux de la collaboration numérique

\section{Formes et enjeux de la collaboration numérique}

\section{Lorna Heaton}

\section{(2) OpenEdition}

\section{Journals}

Édition électronique

URL : http://journals.openedition.org/ticetsociete/1280

DOI : 10.4000/ticetsociete. 1280

Éditeur

Association ARTIC

Référence électronique

Lorna Heaton, «Formes et enjeux de la collaboration numérique », tic\&société [En ligne], Vol. 7, №

1 | 1er semestre 2013, mis en ligne le 04 juin 2013, consulté le 19 avril 2019. URL : http://

journals.openedition.org/ticetsociete/1280 ; DOI : 10.4000/ticetsociete.1280 
tic\&société - 6 (2), $1^{\mathrm{er}}$ semestre 2013

\title{
Formes et enjeux de la collaboration numérique
}

\author{
Lorna HEATON \\ Département de communication \\ Université de Montréal
}

L'arrivée massive des technologies d'information et de la communication a bouleversé les façons de faire dans de nombreux secteurs d'activités, notamment en raison d'une de leurs propriétés essentielles : permettre à des personnes d'horizons différents, séparés dans le temps et l'espace, de collaborer. On a vu ainsi l'émergence de nouvelles formes d'organisations distribuées (ou «en réseau »), avec des équipes de travail autonomes, parfois multinationales. Des plateformes Web sont mobilisées dans le but de coordonner la production médiatique, de solliciter des opinions et de partager des connaissances. Que ce soit de manière formelle ou informelle, des internautes se réunissent et utilisent des ensembles d'outils pour coordonner leurs actions.

Dans la perspective où l'on reconnaît le rôle des dispositifs techniques dans le cadrage des pratiques et la prégnance des conditions matérielles qui les entourent (Law 1987, Star 1989, Suchman 2005), ce numéro a pour but de réfléchir à la relation entre TIC et collaboration. Plus spécifiquement, nous nous arrêtons sur les formes de collaboration qui sont appuyées ou générées par l'utilisation d'outils collaboratifs de type Web 2.0 (O'Reilly 2005). Ces nouvelles plateformes collaboratives ont la particularité d'inciter les internautes ordinaires à participer directement à la création de contenus et à échanger entre eux (Bruns 2008, Jenkins 2006). En canalisant l'interaction et l'échange de contenus entre usagers et la diffusion et rediffusion des contenus, les dispositifs jouent un rôle central de coordination dans les environnements Web 2.0 (Heaton, Millerand et Proulx 2011).

$\mathrm{Si}$ ces outils et plateformes collaboratifs facilitent a priori la collaboration, elles ne la produisent pas à tout coup et peuvent aussi en constituer des obstacles. Dans quelle mesure observe-t-on, ou non, de nouvelles formes de collaboration appuyées sur les technologies de communication? Sommes-nous face à une injonction de collaborer? Dans quelle mesure les relations de coopération médiatisées par les TIC s'avèrent-elles aussi comprendre des rapports de domination? Peuvent-elles contribuer à remettre en cause un ordre 
Formes et enjeux de la collaboration numérique

établi, ou encore favoriser l'émergence d'un nouvel ordonnancement des rapports sociaux? Quels sont les enjeux, notamment en termes de pouvoir ou de contrôle, associés au développement de pratiques dites collaboratives? Dans ce contexte, comment mieux former et préparer les individus à faire face aux défis de la collaboration médiatisée par les TIC? Voilà quelques questions qui ont motivé le choix de ce thème.

En cherchant à poser un regard critique sur la relation entre les TIC et les formes de collaboration contemporaine, l'appel à textes a ratissé large. Ainsi, les textes retenus pour ce numéro couvrent un éventail de sujets. La diversité de provenance disciplinaire des textes reflète la préoccupation pour le sujet de la collaboration dans les domaines de la communication, de la sociologie, des sciences de l'éducation et de la gestion. Cette diversité est reflétée dans la variété des conceptions/définitions de la collaboration ainsi que celle des enjeux soulevés. La plupart des textes ont un ancrage empirique à partir duquel les auteurs proposent une analyse critique des pratiques de collaboration dans leurs usages des TIC. Ils y soulèvent des enjeux de contribution et de participation dans la collaboration en ligne. La métaphore de la conversation (Cardon 2011) est récurrente, tout comme la notion d'activité (Licoppe 2008, Borzeix et Cochoy 2008), de situation (Quéré 1997, Suchman 2008) et du caractère structurant des dispositifs matériels (Leonardi 2010, Heaton et al. 2011). En filigrane, on voit pointer le thème de la visibilité résultant des interrelations entre usagers et dispositifs (Thompson 1995, 2000).

Tous les textes soulèvent les tensions entre le potentiel ou les promesses des plateformes de collaboration et la réalité. Les deux premiers articles (ceux de Ross et de Falgas) s'intéressent à la production et aux producteurs de contenus médiatiques dans les industries créatives et de communication. Un troisième article, de Bonneau, interroge la contribution dans l'entreprise. Dans ces articles, la "conversation " n'est pas dialogique, mais a lieu parfois avec des interlocuteurs inconnus, voire imaginés. Les deux derniers articles proposent des modèles ou principes pour guider un apprentissage critique des outils de collaboration de deuxième génération. Ils réfléchissent explicitement sur le caractère structurant des dispositifs sur la collaboration et dans l'expérience des apprenants.

Philippe Ross développe une conception du rapport producteur-public en tant que « quasi-interaction médiée.» II aborde la production médiatique comme une activité sociale forcément orientée vers un public. Ross soutient que les études 
sur l'interaction humain-ordinateur et sur la médiation tendent à idéaliser l'influence des usagers et en particulier l'interaction directe entre producteur de technologies et « end user ». Cette tendance à donner à l'usager « réel » un rôle actif, sinon déterminant, dans la mise en forme technique est exacerbée par le discours autour des technologies du Web 2.0. Ross invite à reconsidérer le rôle (passif) du public «invoqué » dans la stabilisation de productions médiatiques. Pour ce faire, il reprend le concept de la «quasi-interaction médiée» du théoricien des médias John Thompson (1995). À la différence de Thompson, Ross l'emploie pour penser la communication de masse telle qu'elle est opérée par les producteurs, plutôt que par les récepteurs ou usagers. Cette interaction est une forme d'action à distance qui permet aux individus d'agir au nom d'autrui qui se retrouve dans d'autres espaces et d'autres lieux, ainsi que de réagir aux actions d'individus éloignés. La dynamique n'est pas dialogique, mais unidirectionnelle.

Ross affirme que, si les producteurs sont en mesure d'agir de la sorte, c'est parce qu'ils se basent sur des connaissances tacites, et imparfaites, de leur public. II reprend une «moitié » du « double caractère » de McQuail (1987), pour qui les publics sont constitués à la fois en tant que collectivité formée en réponse aux canaux ou contenus médiatiques, et à partir de forces sociales existant par ailleurs. Dans la relation circulaire entre production et réception, la notion du public aux origines sociales (society origination) suppose que la relation se forme dans des contextes sociaux en amont de la création d'un artéfact ou d'une production particulière, plutôt que par le biais du médium ou de l'artéfact. Ceci permet à Ross d'expliquer comment, en pratique, les producteurs mobilisent ce qu'ils savent du public en amont du contexte de production. En effet, dans une situation d'incertitude quant aux récepteurs/usagers éventuels de leurs artéfacts, les producteurs déploient des stratégies réflexives pour combler le fossé entre connaissances générales et contexte particulier nouveau et incertain. En modélisant l'inconnu sur le familier, ils font preuve d'apprentissage social. Pour agir, ils n'ont pas besoin d'une connaissance parfaite du public anticipé, mais d'une connaissance suffisante. Enfin, Ross conclut qu'un examen de la production en tant qu'activité sociale orientée vers un public jette une lumière sur les artéfacts qui façonnent des formes émergentes de participation comme le réseautage, le contenu généré par les usagers ou le crowdsourcing. Si les producteurs professionnels, seuls, ne peuvent répondre à cette demande forte pour l'information produite par les membres du public «ordinaires », il plaide qu'il est néanmoins important de scruter le travail de ces producteurs au sens traditionnel, car les fruits de leur travail - et leurs visions du public - cadrent la participation des usagers. 
Formes et enjeux de la collaboration numérique

L'article de Julien Falgas décrit la production collaborative d'un bédénovela numérique, Les Autres Gens. Falgas inscrit la production de cette bande dessinée quotidienne dans la continuité de la tradition narrative de la bande dessinée dans laquelle chaque collaborateur est reconnu comme un auteur. Tandis que la production collaborative des produits culturels existe depuis longtemps, Falgas soutient que les nouvelles technologies étendent l'ampleur de cette collaboration et accélère le rythme de production. Le modèle de collaboration illustré ici est centralisé autour du scénariste principal, mais le dispositif y joue un rôle central en prenant en charge une certaine partie du travail de coordination. Falgas mobilise la théorie de l'activité, particulièrement sa «troisième génération» (Engeström 1987), pour illustrer comment les mécanismes de coordination sont constitués dans la pratique (Jarzablowski, Lê et Feldman, 2012). II avance que les collaborateurs de LAG perdent rapidement conscience qu'ils évoluent dans un environnement numérique pourtant indispensable à l'exercice de leur activité et démontre comment cette " automatisation des actions en opérations » facilite la collaboration. Le résultat est une situation de production quasi industrielle, mais qui est vécue par les collaborateurs comme l'activité artistique d'auteur qu'est pour eux la création de bandes dessinées. Dans ce cas-ci, les relations de coopération sont médiatisées par les TIC mais ne remettent pas en cause l'organisation du travail, ni les relations de pouvoir. Cependant, à l'instar de Zuboff (1988) ou Groleau (2008), on pourrait se questionner sur l'impact ultime de l'automatisation sur la conscience réelle qu'ont les collaborateurs de leurs activités de travail.

L'article de Claudine Bonneau, "Travailler à haute voix sur Twitter », étend la notion de la collaboration au-delà d'un petit groupe pour examiner l'utilisation d'un réseau public pour la collaboration informelle. À la différence de Falgas, la collaboration qui l'intéresse n'a pas toujours un but défini et n'est pas circonscrite dans le temps. II s'agit plutôt de la diffusion de messages sur un canal public qui mobilise le caractère « public par défaut » des comptes Twitter. Malgré le caractère public de Twitter, les utilisations qu'explore Bonneau se situent à l'intérieur des organisations, habituellement conçues comme des milieux fermés. Son étude s'inscrit ainsi en porte à faux de la tendance des entreprises à vouloir fournir des réseaux sociaux internes pour la collaboration entre leurs employés.

Bonneau identifie quatre types d'usages de Twitter dans un contexte de travail : la narration du travail en train de se faire (working out loud), le partage des traces de son travail, la diffusion d'intentions et un questionnement lancé à 
la foule anonyme. Elle mobilise le concept d'affordance (Gibson 1979) pour explorer comment les spécificités matérielles et communicationnelles de la plateforme Twitter ouvrent la porte aux utilisations collaboratives. Son texte illustre comment les commentaires et les interactions suscités par ces publications peuvent donner naissance à des réseaux de collaboration informelle, souvent ponctuelle. Dans la mesure où les outils Internet de seconde génération tel que Twitter semblent intégrer différents modèles de visibilité à partir desquels les utilisateurs déplaceraient les frontières entre public et privé, entre « ce qui peut être vu » et « ce qui ne peut être vu » (Cardon 2008), le texte de Bonneau démontre comment cette spécificité contribue concrètement à renouveler les formes d'interaction.

Les pratiques collaboratives décrites par Bonneau soulèvent des questions sur l'ordre établi et le contrôle dans les organisations. Alors que les organisations investissent massivement dans l'implantation d'outils de communication et de collaboration sophistiqués, les employés semblent préférer se servir de canaux de communication non prescrits. Comment les organisations peuvent-elles gérer l'utilisation de plateformes qui rendent les frontières de l'organisation plus perméables, avec les dangers atténuants de fuite d'information et, donc, de perte de contrôle ? De plus, dans la mesure où ces plateformes sont fournies par les employés eux-mêmes, et qu'il s'agit de ressources qu'ils utilisent pour accomplir leur travail, on peut s'interroger sur le sort des employés qui n'y ont pas accès. Se trouvent-ils désavantagés et leur rendement en souffre-t-il ? Avec quelles conséquences organisationnelles ?

Les deux derniers textes de ce numéro adressent spécifiquement les enjeux de l'éducation et de la formation à la collaboration numérique. Le concept d'alphabétisation de réseau (network literacies) attire beaucoup d'attention de la part de chercheurs (Jenkins et al 2009, Rheingold 2010, Lievrouw 2009). Comment et quoi enseigner pour que les usagers ordinaires soient aptes à participer de manière significative? Comment les amener à développer un degré effectif d'expertise en ce qui concerne aussi bien la maîtrise des dispositifs techniques, que la capacité à élaborer des contenus spécifiques, ou encore, la compétence à communiquer dans les environnements numériques. Ce dernier présume la capacité de se situer en relation à d'autres, en plus de comprendre comment l'architecture contraint et habilite l'activité en ligne. Lievrouw (2012) identifie les pédagogies permettant de développer et de renforcer ces compétences comme l'un des enjeux les plus importants de la prochaine décennie. 
Formes et enjeux de la collaboration numérique

C'est dans cet ordre d'idées que Thibaud Hulin propose un modèle de formation centré sur l'autonomie de l'apprenant. Pour lui, le projet de formation à la collaboration numérique appelle une triple compétence : l'apprenant devra comprendre le fonctionnement ou le potentiel des logiciels collaboratifs; il devra réfléchir aux contraintes que font peser sur ses productions les interfaces utilisées; et il devra être apte à réfléchir à ses propres pratiques collaboratives. Son texte décrit un dispositif de formation à l'écriture collaborative numérique conçu dans le but de développer une démarche d'autonomie et de créativité de l'apprenant. Le modèle a été implanté lors d'une formation universitaire qui a été observée puis analysée. L'analyse fait état des avantages de l'intégration de la théorie et d'une démarche réflexive à l'apprentissage: les étudiants ont développé leur compréhension des enjeux de savoir et de pouvoir de tout dispositif numérique en même temps qu'ils apprenaient à utiliser les outils collaboratifs. La contribution de l'article à ce numéro est double : premièrement, l'auteur fournit la critique d'une idéologie de la collaboration qui se limite à « des recettes pour utiliser telle version de telle interface vite dépassée [alors que] la collaboration relève de la communication, et implique des compétences complexes ". Deuxièmement, l'article illustre clairement le caractère contraignant/structurant des dispositifs techniques.

Enfin, Catherine DeLavergne et Marie-Caroline Heïd proposent une réflexion autour de la collaboration numérique à la fois comme moyen et comme finalité dans la formation universitaire des professionnels en communication. Leurs propos sont illustrés par des exemples tirés d'une recherche-action menée depuis 2008 dans le cadre d'un cursus généraliste en Information et Communication dans une université française. Elles insistent sur une orientation vers l'action dans des situations concrètes. DeLavergne et Heïd soutiennent que les compétences collaboratives sont intimement associées à l'évolution des modes de travail et aux transformations des organisations, et que ces évolutions doivent être prises en compte dans la conception d'un dispositif de formation. Ainsi, en plus d'acquérir un ensemble de compétences techniques, les futurs professionnels de la communication devraient être capables d'adapter leurs usages aux diversités des organisations et de comprendre non seulement les implications participatives, mais aussi les logiques prescriptives des dispositifs socio-numériques qu'ils seront appelés à concevoir, à implanter ou à animer. Dans leur souci de s'assurer que les apprenants développent un regard critique sur leurs pratiques professionnelles, ainsi qu'une conscience de l'effet structurant des dispositifs de collaboration, en commençant avec le dispositif de formation, les auteures se positionnent clairement contre une logique instrumentalisée de formation aux TICs collaboratives. Leurs expérimentations 
avec le dispositif de formation tendent à démontrer que rendre visibles et fécondes les tensions permet cette distanciation, tout en répondant aux besoins de formation « du marché » à l'intérieur des prescriptions du cadre universitaire de formation.

Au final, ces cinq articles interrogent, chacun à leur manière, les nouvelles formes de collaboration numérique et les enjeux qui y sont associés. Ils illustrent une variété de situations qui sont perturbées par des outils ou des plateformes collaboratifs, et dans lesquelles l'interaction est médiée, encadrée et canalisée par les dispositifs qui incarnent des visions particulières de la collaboration. Un regard critique sur la relation entre les TIC et les formes de collaboration contemporaine se dégage, regard qui appelle à une attention particulière à la formation non seulement sur l'usage d'outils collaboratifs, mais également sur la compréhension d'enjeux de savoir et de pouvoir pour permettre de vivre «l'injonction à collaborer » tout en faisant preuve d'autonomie et de créativité.

\section{Références bibliographiques}

BORZEIX A. et COCHOY, F., 2008, « Travail et théorie de l'activité : vers des workspace studies ? », Sociologie du travail, 50 (3), pp. 273-286.

BRUNS, A., 2008, Blogs, Wikipedia, Second Life, and Beyond. From Production to Produsage. New York : Peter Lang.

CARDON, D., 2008, « Le design de la visibilité : un essai de cartographie du web 2.0 ", Réseaux no. 152, pp. 93-137.

CARDON, D., 2011, Montrer/regarder: L'économie de la visibilité sur les réseaux sociaux d'Internet. Texte inédit.

ENGESTRÖM, Y., 1987, Learning by expanding: An activity-theoretical approach to developmental research. Helsinki : Orienta-Konsultit.

GIBSON, J. J., 1979, The Ecological Approach to Visual Perception. Boston : Houghton-Mifflin.

GROLEAU, C., 2008, «Integrative Technologies in the Workplace : Using Distributed Cognition to Frame the Challenges Associated with their Implementation ", dans B. Grabot, A. Mayère et I. Bazet (dirs.), ERP Systems and Organisational Change: A Socio-Technical Insight. London : Springer-Verlag, pp. 27-46. 
HEATON, L., MILLERAND, F. et PROULX, S., 2011, « The Role of Collaborative Tools in Making Coordination Sustainable : The Case of TelaBotanica ", 61st Annual Conference of the International Communication Association, Boston, 26-30 mai 2011.

JARZABLOWSKI, P. A., LE, J. K. et FELDMAN, M., 2012, “ Toward a Theory of Coordinating : Creating Coordinating Mechanisms in Practice ", Organization Science, 23(4), pp. 907-927.

JENKINS, H., 2006, Fans, Bloggers and Gamers : Exploring Participatory Culture. New York: NYU Press.

JENKINS, H., avec K. Clinton, R. Purushotma, A.J. Robison et M. Weigel, 2009. Juin, Confronting the Challenges of Participatory Culture: Media Education For the 21st Century. The John D. and Catherine T. MacArthur Foundation Reports on Digital Media and Learning. Cambridge, MA: MIT Press. Disponible à: http://dmlcentral.net/resources/3756

LAW, J., 1987, "Technology and Heterogeneous Engineering: the Case of the Portuguese Expansion ", dans Wiebe E. Bijker, Thomas P. Hughes et Trevor Pinch, (dirs.), The Social Construction of Technological Systems. Cambridge MA: MIT Press, pp. 111-134.

LEONARDI, P.M., 2010, « Digital materiality? How artifacts without matter, matter », First Monday, vol. 15, no 6.

LICOPPE, C., 2008, "Dans « le carré de l'activité »: perspectives internationales sur le travail et l'activité », Sociologie du travail, 50, pp. 287302.

LIEVROUW, L.A., 2009, « The uses of disenchantment in new media pedagogy: Teaching for remediation and reconfiguration ", dans R. Hammer et D. Kellner (dirs.), Media/Cultural Studies: Critical Approaches. New York: Peter Lang, pp.560-575.

LIEVROUW, L.A., 2012, "The Next Decade in Internet Time: Ways Ahead for New Media Studies », Information, Communication and Society, 15(5), pp. 616-638.

McQUAIL, D., 1987, Mass communication theory: An introduction (second edition). London: Sage.

O'REILLY, T., 2005, Qu'est ce que le web 2.0 : Modèles de conception et d'affaires pour la prochaine génération de logiciels http://www.internetactu.net/2006/04/21/quest-ce-que-le-web-20-modeles-deconception-et-daffaires-pour-la-prochaine-generation-de-logiciels/ 
QUÉRÉ, L., «1997, « La situation toujours négligée ? », Réseaux, vol. 15, no 85, pp. 163-192.

RHEINGOLD, H., 2010, « Attention, and other 21st century social media literacies ", EDUCAUSE Review,45(5), September/October, pp. 14-24. Disponible à: http://www.educause.edu/EDUCAUSE+Review/EDUCAUSEReviewMagazin eVolume45/AttentionandOther21stCenturySo/213922

STAR, S. L., 1989, " The Structure of III-Structured Solutions: Boundary Objects and Heterogeneous Distributed Problem Solving », Dans M. Huhns et L. Gasser, éds. Readings in Distributed Artificial Intelligence. Menlo Park, CA: Morgan Kaufman, pp. 37-54.

SUCHMAN, L., 2008, Human-Machine Reconfigurations: Plans and Situated Actions, $\left(2^{\mathrm{e}}\right.$ éd) Cambridge : Cambridge University Press.

SUCHMAN, L., 2005, «Affiliative Objects », Organization, 12(3), pp. 379-399.

THOMPSON, J. B., 1995, The Media and Modernity. A Social Theory of the Media. Cambridge: Polity Press.

THOMPSON, J. B., 2000, “Transformation de la visibilité », Réseaux, 100, pp. 187-213.

ZUBOFF, S., 1988, In the Age of the Smart Machine: The Future of Work and Power. New York : Basic. 